\title{
Contextual-specificity of short-delay extinction in humans: Renewal of fear-potentiated startle in a virtual environment
}

\author{
Ruben P. Alvarez, ${ }^{1}$ Linda Johnson, and Christian Grillon \\ Mood \& Anxiety Disorders Program, National Institute of Mental Health, NIH, Bethesda, Maryland 20892, USA
}

\begin{abstract}
A recent fear-potentiated startle study in rodents suggested that extinction was not context dependent when extinction was conducted after a short delay following acquisition, suggesting that extinction can lead to erasure of fear learning in some circumstances. The main objective of this study was to attempt to replicate these findings in humans by examining the context specificity of short-delay extinction in an $A B A$ renewal procedure using virtual reality environments. A second objective was to examine whether renewal, if any, would be influenced by context conditioning. Subjects underwent differential aversive conditioning in virtual context $A$, which was immediately followed by extinction in virtual context B. Extinction was followed by tests of renewal in context A and B, with the order counterbalanced across subjects. Results showed that extinction was context dependent. Evidence for renewal was established using fear-potentiated startle as well as skin conductance and fear ratings. In addition, although contextual anxiety was greater in the acquisition context than in the extinction context during renewal, as assessed with startle, context conditioning did not influence the renewal effect. These data do not support the view that extinction conducted shortly after acquisition is context independent. Hence, they do not provide evidence that extinction can lead to erasure of a fear memory established via Pavlovian conditioning.
\end{abstract}

Fear conditioning has long been proposed to be involved in the etiology and maintenance of anxiety disorders (Mineka and Zinbarg 1996; Bouton et al. 2001). According to this view, an initially neutral conditional stimulus (CS) becomes an elicitor of fear after being associated with an aversive event or unconditioned stimulus (US). Exposure therapy, one of the most effective treatments for anxiety disorders, attempts to counteract the CSUS association through repeated and systematic confrontation with fear-provoking stimuli. Repeated confrontation with the CS in the absence of the US leads to extinction, a reduction or loss in conditioned fear responses. Because suppressing powerful emotional and traumatic memories is likely to be crucial to successful treatment of anxiety disorders, there is growing interest in better understanding the mechanisms that underlie extinction (Quirk 2002; Bouton 2004; Davis et al. 2006).

While exposure therapy is effective in reducing fear (see Craske et al. 1997; Ost et al. 1997), therapy is frequently followed by a return of fear with the passage of time (Rachman and Lopatke 1988). This clinical observation is consistent with empirical evidence from preclinical studies that demonstrate that the acquisition of conditioned fear is robust and context independent but that extinction is weak and context dependent (Bouton 2004). The fragility of fear suppression may be due to the fact that extinction does not cause unlearning or forgetting of the CS-US association. It is generally agreed that extinction does not erase original fear learning; rather, it creates a new learning that competes with the original fear memory. Evidence supporting the hypothesis that extinction leads to new learning comes from the observation that fear recovers spontaneously following the passage of time, and from studies of renewal and reinstatement that show that extinction is context specific.

In a renewal experiment, acquisition of fear is conducted in a given context (A) and extinction takes place in another context

\footnotetext{
${ }^{1}$ Corresponding author.
}

E-mail alvarezr@mail.nih.gov; fax (301) 594-9959.

Article is online at http://www.learnmem.org/cgi/doi/10.1101//m.493707.
(B). The test that determines retention of extinction learning is carried out in the original acquisition context (ABA design), in the extinction context (ABB design), or in a new context (ABC design). Under these conditions, return of fear is seen in the original acquisition context (A) and in the new context $(\mathrm{C})$ but not in the extinction context (B). In reinstatement, fear returns to the CS when an unsignaled US is presented after extinction. Reinstatement is also context dependent: Fear reemerges only when exposure to the US and the reinstatement test occur in the same context. The context specificity of extinction has been repeatedly demonstrated in animal studies (Bouton 2004), and similar results have recently emerged from the human literature (LaBar and Phelps 2005; Milad et al. 2005; Vansteenwegen et al. 2005; Neumann 2006).

While the "new learning" hypothesis of extinction appears to be supported by strong empirical evidence, new data recently challenged this view. Myers et al. (2006) reported results in rodents consistent with the hypothesis that extinction can erase the memory of the fear response (i.e., the CS-US association). Myers et al.'s contention was based on the observation that under some circumstances extinction is not context dependent. More specifically, using renewal and reinstatement tests, these investigators replicated the context specificity of extinction when acquisition and extinction were conducted $72 \mathrm{~h}$ apart but not when extinction was conducted shortly after acquisition. For example, in their ABA renewal test, rats that underwent acquisition in context $A$ and extinction in context $B$ showed a return of fear in context $\mathrm{B}$ when the time interval between acquisition and extinction was $72 \mathrm{~h}$ but not when it was $10 \mathrm{~min}$ or $1 \mathrm{~h}$. Myers et al. argued that "new learning" and "unlearning" mechanisms of extinction were two potential outcomes of different experimental conditions, with the critical variable being the time interval between acquisition and extinction. They further pointed out that the hypothesis of multiple mechanisms of extinction was consistent with recent reports showing that the neural mechanisms of extinction vary when extinction is conducted immediately versus a few hours following acquisition (Cain et al. 2005). 
The "unlearning" hypothesis of extinction relies on the observation that when extinction is conducted shortly after acquisition, extinction is not context specific. While these results may be consistent with the animal literature, they do not seem consistent with research in humans. Indeed, several studies in humans have now reported context specificity of extinction when extinction is conducted immediately after acquisition using renewal and reinstatement tests.

However, differences in experimental conditions between studies in humans and in animals could potentially explain the discrepancy in the results. One important difference is the measure of conditioned fear responses used in the studies. Myers et al. (2006) measured conditioned fear using fear-potentiated startle. Fear-potentiated startle refers to the increase in startle reactivity when elicited in the presence of a CS that has been previously paired with an aversive US. Fear-potentiated startle is a reliable and objective measure of fear in both animals and humans (Davis 1986; Grillon and Baas 2003). It is influenced by limbic structures known to play a crucial role in fear and anxiety (Walker et al. 2003). In humans, evidence for return of fear has been reported using behavioral (reaction time task reflecting allocation of attention to the CS) (Hermans et al. 2005), verbal (ratings of fear and US expectancy) (Vansteenwegen et al. 2005; Neumann et al. 2007), and psychophysiological (skin conductance response) (LaBar and Phelps 2005; Milad et al. 2005; Vansteenwegen et al. 2005) indices of fear. All of these measures are indirect measures of fear (Grillon and Baas 2003). Verbal ratings are vulnerable to individual perception, demand characteristics, and intentional distortion. The skin conductance response is a nonspecific measure of arousal that reflects orienting to a stimulus as a function of its relevance and not necessarily its emotional significance (Dawson and Furedy 1976; Hamm and Vaitl 1996). So far, no study has investigated the contextual control of extinction in humans using fear-potentiated startle.

Because procedures that may lead to an unlearning of conditioned fear memories hold promise for clinical application, it is critical to explore the generality of the findings by Myers et al. (2006) to human behavior. A translational approach to extinction mechanisms is promoted by increasing the commonalities between human and animal studies (Branch and Hackenberg 1998). Therefore, the main objective of the present study was to examine the return of fear in humans when extinction was conducted immediately after acquisition using fear-potentiated startle. Other indices of fear were also used to relate our findings to the literature in humans.

Animal and human studies also differ with regard to the nature of the contextual stimuli used. Rodents are placed in different physical contexts (i.e., cages), while in some human studies, contextual modulation was implemented by merely changing the background stimuli. In the present study, we used computer-generated virtual reality (VR) to examine the impact of changes in virtual contexts, which may approximate more closely the physical contexts used in animal studies. Prior research in our laboratory has validated VR to study context conditioning and has demonstrated that computer-generated VR contexts overshadow the surrounding physical context (i.e., the experimental room) (Grillon et al. 2006).

A secondary aim of the study ad- dressed the issue of the nature of the contextual control of extinction in an ABA renewal test. One possibility is that renewal is promoted by excitatory associations from contextual cues in context A that summate with undetected associative strength (or fear) remaining in extinguished CS (Bouton and King 1983). Bouton and King (1983) did not find support for this hypothesis in animal studies. They showed that ABA renewal can occur in the absence of any contextual fear present during renewal testing in context A. As an alternative, Bouton (2004) proposed that following acquisition in $\mathrm{A}$ and subsequent extinction in $\mathrm{B}$, the CS is ambiguous. Bouton (2004) suggested that the contexts help retrieve the relation between the CS and the US in a given context, a process sometimes referred to as occasion setting (see Holland 1992). In other words, the context clarifies the meaning of the ambiguous CS. In the present study, we used the startle reflex to assess contextual anxiety present in the acquisition and extinction contexts during renewal. It was expected that contextual anxiety would not affect renewal.

\section{Results}

\section{Startle}

\section{Acquisition and extinction}

A schematic of the experiment and pictures of the virtual environments used as contexts are displayed in the top and bottom panels of Figure 1. An ABA design was used in which subjects were conditioned in context $\mathrm{A}$, extinguished in context $\mathrm{B}$, and returned to contexts $\mathrm{A}$ and $\mathrm{B}$ for a renewal test. The experiment consisted of three phases with a brief break between each phase. During each phase, a prerecorded VR scenario was presented (for details, see Materials and Methods). The first phase consisted of the first acquisition period in context A. The second phase consisted of the second acquisition period in context A and two extinction periods in context $\mathrm{B}$. The third phase consisted of a third extinction period in context $\mathrm{B}$ and a renewal test in contexts A and B. During each acquisition period, each CS was presented four times for a total of eight CS+ and eight CS - . Nine startle stimuli (three $\mathrm{CS}+$ /three $\mathrm{CS}-/$ three intertrial intervals [ITI]) were delivered during the second acquisition period to assess fear conditioning. During each extinction period, each CS was presented six times for a total of $18 \mathrm{CS}+$ and $18 \mathrm{CS}-$, but only 15 startle stimuli (five CS+/five CS -/five ITI) were deliv-

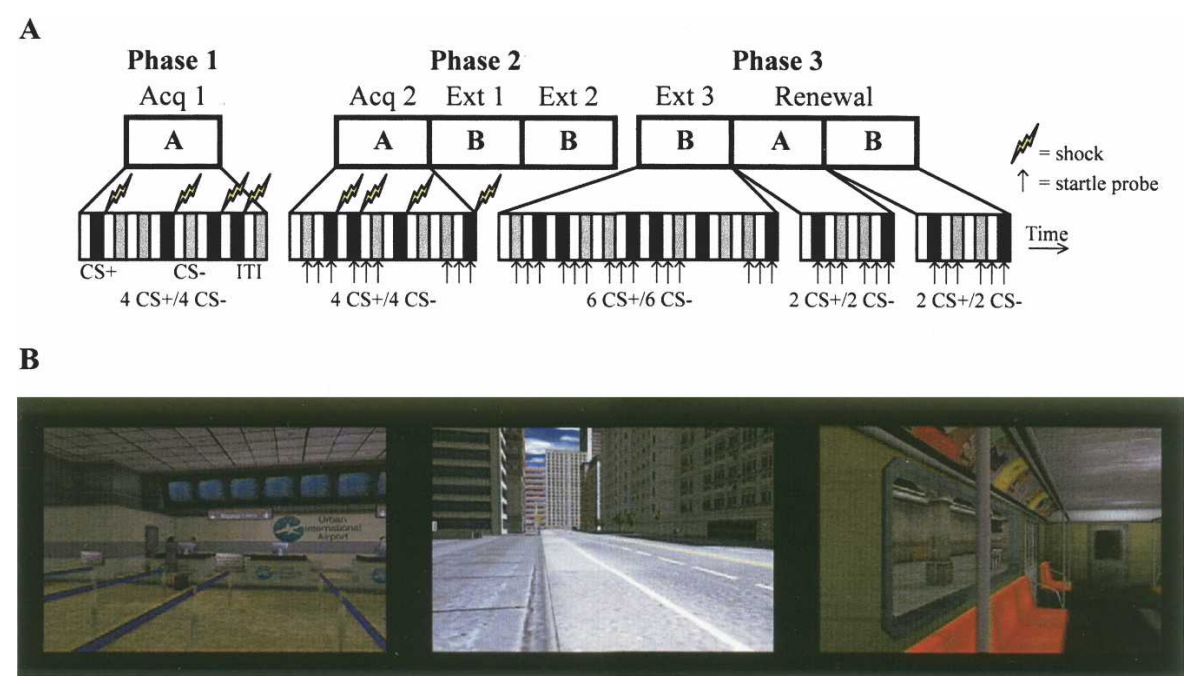

Figure 1. (A) Experimental protocol. Black, gray, and white bars represent $\mathrm{CS}+, \mathrm{CS}-$, and ITI, respectively. $(B)$ Pictures of the three virtual environments: the airport, the city street, and the subway. 
ered. Therefore, the data were averaged over three successive trials resulting in five extinction blocks (Ext 1-Ext 5). During the renewal test in each context, two CS+ and two CS - were presented, and six startle stimuli (two CS+/two CS - /two ITI) were delivered.

The startle results are shown in Figure 2. Subjects conditioned successfully in the acquisition context. Conditioning generalized to the extinction context early on and then progressively extinguished as the CS+ was continuously presented without the shock. This pattern of response was supported by a significant Stimulus Type $\times$ Block interaction $\left(F_{(5,75)}=2.6, P<0.03\right)$ and a linear Stimulus Type $\times$ Block interaction $\left(F_{(1,15)}=8.5, P<0.01\right)$. Follow-up tests confirmed that startle was larger during CS+ compared to CS - in the acquisition context $\left(F_{(1,15)}=33.3\right.$, $P<0.0001)$ and early in the extinction context during extinction blocks 1 ( $\left.F_{(1,15)}=7.0, P<0.02\right), 2\left(F_{(1,15)}=22.1, P<0.0009\right)$, and $3\left(F_{(1,15)}=10.2, P<0.006\right)$. There was no longer differential responding to the two CSs in blocks $4\left(F_{(1,15)}=2.8, P=\right.$ ns [not significant $]$ ) and $5\left(F_{(1,15)}=1.4, P=\mathrm{ns}\right)$, indicating that the fear to the CS+ extinguished.

\section{Renewal test}

The conditioned response reemerged fully when the subjects returned to the acquisition context but remained extinguished when they returned to the extinction context (Stimulus Type $\times$ Context: $\left.F_{(1,15)}=8.5, P<0.01\right)$. Follow-up tests showed greater startle to the CS+ compared with the CS - in the acquisition context $\left(F_{(1,15)}=41.7, P<0.0001\right)$ but not in the extinction context $\left(F_{(1,15)}=2.5, P=\mathrm{ns}\right)$. The magnitude of conditioned responses in the acquisition context during renewal was similar to that in the initial acquisition context (10 T score vs. $8.9 \mathrm{~T}$ score, respectively: $F_{(1,15)}=0.3, P=$ ns).

To examine the possibility that renewal in the acquisition context resulted from an incomplete extinction of conditioned responses, we compared renewal in subjects who had fast extinction with subjects who had slow extinction. We calculated the mean startle responses for CS+ and CS - over the last three extinction blocks (average of nine CS+ and nine CS -) and calculated the difference scores between $\mathrm{CS}+$ minus $\mathrm{CS}-$. Subjects were divided into a fast extinction group and a slow extinction group based on a median split of these scores (Table 1). As expected, based on the last three extinction blocks, the two groups showed different responses to the CS+ and CS-. A Group $\times$ Stimulus Type ANOVA on the extinction data revealed a significant Group $\times$ Stimulus Type interaction $\left(F_{(1,14)}=39.3\right.$, $P<0.0001)$. The slow extinction group showed greater startle during CS+ compared with CS $-\left(F_{(1,7)}=69.7, P<0.0001\right)$, while the fast extinction group did not show such a difference $\left(F_{(1,7)}=1.1, P=\mathrm{ns}\right)$. Despite this difference in extinction, the two

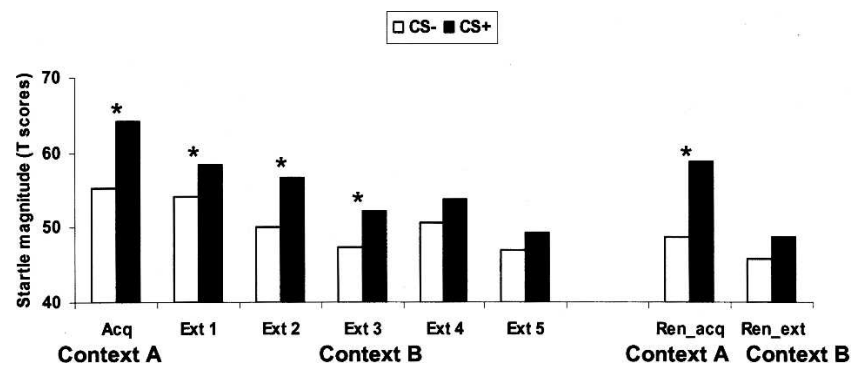

Figure 2. Magnitude of the startle reflex elicited during $C S+$ and $C S-$ during acquisition (context $A$ ), extinction (context $B$ ), and renewal test in the acquisition and extinction contexts. Note that there were only five blocks (instead of six blocks) of three trials per block because only 15 of the 18 trials per CS contained a startle probe. ${ }^{*} P<0.05$. groups showed similar renewal (Stimulus Type $\times$ Extinction Group interaction during renewal: $F_{(1,14)}=1.3, P=$ ns).

\section{Effect of contextual anxiety}

Contextual anxiety was operationally defined as the magnitude of startle during ITI (Grillon and Davis 1997; Baas et al. 2004). The magnitude of ITI startle during renewal was significantly larger in the acquisition context $($ mean $=44.5 ; \mathrm{SEM}=1.5 \mathrm{~T}$ scores) compared with the extinction context (mean $=39.8$; SEM $=0.7$ T scores; $F_{(1,15)}=5.2, P<0.04$ ), suggesting greater contextual anxiety in the acquisition context. The fact that contextual anxiety during renewal was greater in the acquisition context compared with the extinction context does not necessarily mean that contextual anxiety mediated renewal in the acquisition context. To investigate the effect of contextual anxiety on renewal, we compared renewal in subjects who showed substantial contextual anxiety to those who showed little or no contextual anxiety. We calculated the difference scores between startle magnitudes during ITI in the acquisition context minus startle magnitudes during ITI in the extinction context. Subjects were divided into a contextual anxiety group and a no contextual anxiety group based on a median split of these scores (Table 2). Both the contextual anxiety group and the no contextual anxiety group showed greater startle to CS+ compared with CS - in the acquisition context (renewal effect: $F_{(1,7)}=10.3, P<0.01$ and $F_{(1,7)}=61.1, P<0.0001$, respectively), but not in the extinction context. In fact, renewal was nonsignificantly greater in the no contextual anxiety group compared with the contextual anxiety group, which is in the opposite direction to prediction based on a context conditioning account of renewal.

\section{Skin conductance response}

\section{Acquisition and extinction}

The skin conductance response (SCR) results are shown in Figure 3. Subjects conditioned successfully in the acquisition context, and conditioned responses transferred to the extinction context before extinguishing rapidly. This pattern of response was supported by a significant Stimulus Type main effect $\left(F_{(1,15)}=8.1\right.$, $P<0.01)$ and a Stimulus Type $\times$ Block interaction $\left(F_{(6,90)}=5.2\right.$, $P<0.002, e=0.57)$. Follow-up tests confirmed that SCR was larger during CS+ compared with CS - in the acquisition context $\left(F_{(1,15)}=14.3, P<0.002\right)$, and during the first extinction block $\left(F_{(1,15)}=4.9, P<0.04\right)$, but not subsequently (all $\left.P>0.1\right)$.

\section{Renewal}

As with startle, the conditioned response reemerged fully when subjects returned to the acquisition context but remained extinguished when they returned to the extinction context (Stimulus Type $\times$ Context: $\left.F_{(1,15)}=6.6, P<0.02\right)$. Subsequent tests showed greater SCR to the CS+ compared with the CS - in the acquisition context $\left(F_{(1,15)}=9.0, P<0.009\right)$ but not in the extinction context $\left(F_{(1,15)}=0.4, P=\mathrm{ns}\right)$. The magnitude of the conditioned response in the acquisition context during renewal was not as large as in the initial acquisition context $(3.1 \log \mu \mathrm{S}$ vs. $4.7 \log \mu \mathrm{S}$, respectively), but this difference did not reach significance $\left(F_{(1,15)}=1.5\right.$, $P=\mathrm{ns})$.

\section{Subjective anxiety}

Subjective reports of anxiety were taken only after the renewal test. Subjects rated the two CSs in the acquisition and extinction contexts of the renewal test differently $\left(F_{(1,15)}=15.1, P<0.0001\right)$. In the acquisition context, the CS+ was more anxiogenic than the CS - (mean (SEM) 4.7 [0.5] vs. 1.7 [0.2], respectively: $\left.F_{(1,15)}=26.7, P<0.0001\right)$, but in the extinction context, the CS+ and CS - did not differ significantly (1.8 [0.3] vs. 2.0 [0.3], re- 
Table 1. Startle magnitude (SEM) during $\mathrm{CS}+/ \mathrm{CS}$ - during extinction (averaged over last three extinction blocks) and during renewal in subjects with fast and slow extinction

\begin{tabular}{|c|c|c|c|c|c|c|}
\hline \multirow[b]{3}{*}{ Group } & \multirow{2}{*}{\multicolumn{2}{|c|}{$\begin{array}{c}\text { Extinction } \\
\text { Extinction context }\end{array}$}} & \multicolumn{4}{|c|}{ Renewal } \\
\hline & & & \multicolumn{2}{|c|}{ Acquisition context } & \multicolumn{2}{|c|}{ Extinction context } \\
\hline & $\mathrm{CS}_{+}$ & CS- & $\mathrm{CS}_{+}$ & CS- & $\mathrm{CS}_{+}$ & CS - \\
\hline Fast extinction $(N=8)$ & $48.5(0.7)$ & $49.7(0.9)$ & $60.8(2.0)$ & $50.9(2.0)^{\star *}$ & $48.0(2.3)$ & $47.9(1.6)$ \\
\hline Slow extinction $(N=8)$ & $55.0(0.7)$ & $47.0(0.9)^{\star * *}$ & $56.8(2.0)$ & $46.8(2.0)^{* *}$ & $49.7(2.3)$ & $43.9(1.6)$ \\
\hline
\end{tabular}

Asterisks indicate startle during $\mathrm{CS}+>$ startle during $\mathrm{CS}-$ at ${ }^{* *} P<0.01,{ }^{* \star *} P<0.001$.

spectively: $\left.F_{(1,15)}=0.2, P=\mathrm{ns}\right)$. The two contexts did not generate different levels of anxiety (1.8 [0.5] vs. $1.3[0.6]$, respectively: $\left.F_{(1,15)}=0.4, P=\mathrm{ns}\right)$.

\section{Discussion}

This study investigated the contextual control of extinction in an ABA design. There is little disagreement in the literature as to whether extinction is context specific when there is a relatively long period $(>24 \mathrm{~h}$ ) between acquisition and extinction. The lack of contextual-specificity of extinction in rodents has been reported recently when extinction is conducted shortly after acquisition, suggesting that extinction can erase the memory of the fear learning in some circumstances (Myers et al. 2006). In that study, extinction generalized across the extinction and acquisition contexts in an ABA design when extinction was conducted $10 \mathrm{~min}$ or $1 \mathrm{~h}$ after acquisition. The present results, also based on an ABA renewal design, are not consistent with this view. Memory for extinguished fear memory was context specific. During renewal, memory for extinguished fear memory to the CS+ was preserved in the extinction context, but conditioned fear reemerged in the original conditioning context following extinction immediately after acquisition. The most straightforward interpretation of our data is that they do not support the view that extinction leads to an erasure of the original fear learning in humans. Rather, it is consistent with the hypothesis that extinction leaves the original fear learning intact, but adds a new meaning to the CS, and that the two meanings of the CS can be clarified by the context (Bouton 2004).

Consistent with prior studies in humans using verbal report and the skin conductance response (LaBar and Phelps 2005; Milad et al. 2005; Vansteenwegen et al. 2005), the present results found renewal of fear after extinction in an ABA design. In humans, renewal effects have been reported when extinction was conducted either immediately or $>24 \mathrm{~h}$ after acquisition (LaBar and Phelps 2005; Milad et al. 2005; Vansteenwegen et al. 2005). The present study extends these results using an established measure of fear and anxiety, the startle reflex. The startle reflex is considered a translational tool to investigate fear and anxiety because it is a cross-species measure modulated by the amygdala (Grillon and Baas 2003). It is noteworthy that the hypothesis that extinction is not always under contextual control is based on findings using the startle reflex (Myers et al. 2006). Therefore, it is unlikely that our failure to replicate Myers et al's findings can be attributed to our measure of fear conditioning. An important paradigmatic difference between the two studies is the number of extinction trials. Myers et al. (2006) used 90 extinction trials, whereas we used only 18 trials. This raises the possibility that our procedure may have led to incomplete or fragile extinction, which caused conditioned fear to reemerge in the acquisition context during renewal. However, this hypothesis is unlikely. First, startle magnitude during CS+ and CS - were no longer significant by the fourth extinction block, and a fifth extinction block was conducted. Second, renewal was similar among subjects who extinguished rapidly and subjects who extinguished more slowly (see Table 1). One would expect less renewal in the slow extinction group if incomplete extinction affected renewal. Third, if extinction was incomplete, one could have expected renewal in the extinction context. In fact, during pilot studies with less extinction trials, we obtained renewal in the extinction context despite evidence of no differential response to the CS+ and CS - at the end of extinction. Fourth, the number of extinction trials (18) relative to the number of acquisition trials (eight) was comparable to that of other human studies that found renewal (LaBar and Phelps 2005; Milad et al. 2005; Vansteenwegen et al. 2005).

A potential reason for not replicating the finding of Myers et al. (2006) may be due to other characteristics of the study. For example, Myers and colleagues used a single cue conditioning procedure, whereas we used differential conditioning. It is unclear how single versus differential conditioning could affect renewal differently. One hypothesis is that extinction learning may proceed differently in single cue and differential conditioning, with the latter being context dependent and the former not. Inconsistent with this hypothesis, a recent human study using single cue conditioning found context-dependent reinstatement after eight extinction trials subsequent to acquisition with four trials (LaBar and Phelps 2005). This finding indicates that the contextual specificity of extinction is detectable using single cue conditioning and relatively few extinction trials. Another difference between the two studies is that we tested retention of extinction immediately after the final extinction period, whereas

Table 2. Startle magnitude (SEM) during ITI, CS+, and CS - during renewal in subjects with substantial contextual anxiety and with little or no contextual anxiety

\begin{tabular}{|c|c|c|c|c|c|c|}
\hline \multirow[b]{3}{*}{ Group } & \multicolumn{6}{|c|}{ Renewal } \\
\hline & \multicolumn{3}{|c|}{ Acquisition context } & \multicolumn{3}{|c|}{ Extinction context } \\
\hline & ITI & $\mathrm{CS}_{+}$ & CS- & ITI & $\mathrm{CS}_{+}$ & CS- \\
\hline No contextual anxiety & $40.6(1.6)$ & $59.4(1.7)$ & $46.2(1.4)^{\star \star \star}$ & $41.5(0.6)$ & $48.4(2.2)$ & $46.2(1.8)$ \\
\hline Contextual anxiety & $48.4(1.8)^{*}$ & $58.2(2.4)$ & $51.4(2.3)^{\star *}$ & $38.0(0.8)$ & $49.2(2.5)$ & $44.8(1.7)$ \\
\hline
\end{tabular}

Asterisks indicate ITI startle during acquisition context $>$ ITI startle during extinction context or startle during CS+ $>$ startle during CS - at $* P<0.05$, ${ }^{* *} P<0.01,{ }^{* * *} P<0.001$. 


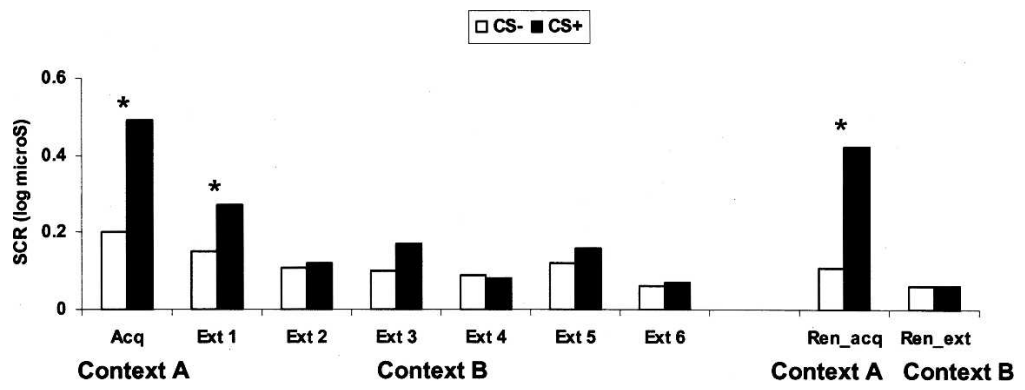

Figure 3. Skin conductance response (SCR) to the onset of the $C S+$ and $C S$ - during acquisition (context A), extinction (context $\mathrm{B}$ ), and renewal test in the acquisition and extinction contexts. ${ }^{*} P<0.05$.

Myers et al. (2006) conducted a post-extinction test $24 \mathrm{~h}$ after extinction. It could be argued that we found renewal of fear following extinction because we used a short-term retention test, whereas Myers et al. (2006) failed to find such a reemergence of fear because they used a long-term retention test. This seems unlikely because a recent study of freezing in animals reported that short-delay extinction led to robust spontaneous recovery of fear in the conditioning context following a long-term $(24 \mathrm{~h})$ retention interval (Maren and Chang 2006). Because spontaneous recovery and renewal are both thought to reflect the context dependence of extinction (Bouton 2004), it is unlikely that renewal would be affected differently by long-term retention. However since renewal was assessed in the present study only after a short-term retention interval, this remains an empirical question. Although our study and that of Maren and Chang (2006) used different retention intervals, each demonstrates that extinction was not due to the erasure of fear memory despite administering extinction shortly after acquisition. To reconcile their results with those of Davis and colleagues (Myers et al. 2006), Maren and Chang (2006) proposed that retention of extinction may depend on an interaction between the interval between acquisition and extinction and the intensity of the US. They suggest that short-delay extinction may be optimal (i.e., enduring and insensitive to context shifts) only following conditioning with weak and non-traumatic US, not with strong and traumatic US, implying that Myers et al. (2006) found erasure of conditioned fear memory following short-delay extinction because they used weak non-traumatic shocks. Reversed, this view could be used to argue that we did not find evidence for memory erasure because we used strong traumatic shocks. This interpretation is highly unlikely because we used only mildly unpleasant shocks that are ethical and unlikely to be experienced as traumatic. Finally, another obvious possibility for the failure to replicate the findings of Myers et al. is that there are species-specific characteristics in the contextual modulation of extinction. To explore this hypothesis, future studies should examine renewal in nonhuman primates.

A secondary aim of the study was to examine the influence of context conditioning on renewal. One possibility is that renewal is promoted by excitatory associations from contextual cues in context A that summate with undetected associative strength (or fear) that remains in the extinguished CS (Bouton and King 1983). We investigated context conditioning using startle magnitude during ITI. We have previously shown that ITI startle magnitude is a sensitive measure of contextual anxiety in real and in virtual contexts (Grillon and Davis 1997; Grillon et al. 2006). We reported that greater ITI startle magnitude in one context compared with another context is also associated with contextual avoidance of the former context relative to the second (Grillon et al. 2006). In the present study, startle magnitude dur- ing ITI was greater in the acquisition context compared with the extinction context during renewal, suggesting greater contextual anxiety in the acquisition context. Despite this difference in contextual anxiety between the two contexts during renewal, contextual anxiety did not seem to affect renewal. Renewal did not differ in subjects who showed no contextual anxiety compared with subjects who showed substantial contextual anxiety (see Table 2 ). If contextual anxiety summated with the remaining fear to the CS after extinction, one would have expected greater renewal in subjects showing more contextual anxiety. Bouton (2004) has proposed that the most likely explanation for renewal is that features of the contexts serve to disambiguate the CS. Thus, the renewal context helps to recover the latent association of the CS with the US.

The present finding that renewal was not dependent on the associative status of the acquisition context (i.e., the increased contextual anxiety in the acquisition context relative to the extinction context) is consistent with the emerging literature on protection from extinction. Recent studies of this phenomenon indicate that concurrent stimuli presented during extinction (e.g., CS) may function like the extinction context in a renewal design, and gate the inhibitory learning that occurs during extinction (Lovibond et al. 2000; Hermans et al. 2006; Vervliet et al. 2007). Whereas return of fear occurs when a CS is encountered in the absence of the extinction context in renewal, fear returns in the absence of a concurrent CS in protection from extinction. In human studies, it seems that the degree to which fear returns when a concurrent CS is omitted (i.e., the amount of protection from extinction that occurs) is independent of whether the concurrent CS was previously established as inhibitory or excitatory. This is similar to our finding, and other data, showing that renewal is independent of the associative status of the context, and suggests that a similar mechanism may underlie protection from extinction and renewal.

Renewal was quite strong with all our measures of conditioning. One important factor in renewal is the degree of contextual change; the more dissimilar the contexts, the greater renewal (Thomas et al. 2003). Contextual manipulations in animals usually rely on changes in the physical context (e.g., the cage) along with accompanying changes in sensory information (e.g., olfactory stimuli). In humans, contextual changes rarely involve the physical context (LaBar and Phelps 2005). More frequently, human fear conditioning studies have relied on modification of background two-dimensional visual stimuli (Milad et al. 2005) or changes in background illumination (Vansteenwegen et al. 2005). In the present study, the use of dramatically different virtual contexts may have promoted robust renewal. As the present and past (Baas et al. 2003; Grillon et al. 2006) studies demonstrate, computer-generated VR is an effective tool for exploring contextual modulation of conditioned responses. One important advantage of VR is that it permits the development of well-controlled procedures in within-subjects designs. In addition, VR enables subjects to experience distinct contexts while remaining stationary in the laboratory. This raises the question of whether the experimental context more generally (i.e., the room where the experiment is actually conducted) modulated or interfered with conditioned responses. While it is possible that context conditioning develops to the experimental room, this does not seem to interfere with conditioned performance in the VR contexts. Because renewal could be tested in the virtual ex- 
tinction and acquisition context, any effect due to context conditioning to the experimental room was controlled for in this study. In addition, we have evidence that context conditioning to virtual contexts overshadows to a great extent the context of the experimental room. In our previous study, we found that conditioned responses obtained in the virtual environment were preserved when the subjects were moved to a new room and re-exposed to the virtual contexts (Grillon et al. 2006).

In summary, the present results show clear evidence of contextual modulation of an extinguished conditioned fear response measured with the startle reflex, the SCR, and verbal report. These results support the hypothesis that extinction does not erase fear memory in humans, even when extinction is initiated shortly after acquisition. Our findings also suggest that fear renewal is not caused by contextual anxiety. As proposed by Bouton (2004), contextual cues may help disambiguate the meaning of the CS. Combining VR and brain imaging may help uncover the neural structures that are involved in occasion setting or retrieving the relation between the CS and the US in a given context.

\section{Materials and Methods}

Participants were 16 healthy volunteers, eight females and eight males (mean age $=26.7 \mathrm{yr}, \mathrm{SD}=7.8 \mathrm{yr}$ ) who gave written informed consent approved by the NIMH Human Investigation Review Board. Inclusion criteria included (1) no past or current psychiatric disorders as per Structured Clinical Interview for DSM-IV (First et al. 1995), (2) no medical condition that interfered with the objectives of the study as established by a physician (e.g., tachycardia), and (3) no use of illicit drugs or psychoactive medications as per urine screen.

\section{Stimuli and apparatus}

\section{VR environment}

The software application (VR Worlds, Psychology Software Tools, Inc.) consisted of several interconnected virtual environments. Three of these environments, an airport, a city street, and a subway, were the contexts used in the present study (see Fig. 1). The airport and city street were separated by the subway system, which was used to travel between contexts.

\section{Psychophysiology system}

Stimulation and recording were controlled by a commercial system (Contact Precision Instruments). The physiological measures recorded were electromyography (EMG) and skin conductance. The acoustic startle stimulus was a $40 \mathrm{msec}$ duration, 103-dB (A) burst of white noise with a near instantaneous rise time presented binaurally through headphones. The startle reflex was recorded with two 6-mm tin electrodes placed under the left eye. Amplifier bandwidth was set to $30-500 \mathrm{~Hz}$ with a sampling rate of $1000 \mathrm{~Hz}$. Electric shocks (up to $5 \mathrm{~mA}$ and $100 \mathrm{msec}$ duration) were produced by a constant current stimulator and administered on the left wrist. The left palmar skin conductance was recorded on the index and middle finger of the left hand according to published recommendations (Prokasy and Ebel 1967).

\section{Design}

Navigation in each environment was prerecorded, edited, and rendered as digitized scenarios; therefore, subjects had no control of events during the experiment and passively observed each VR scenario. Subjects transitioned between the airport and the city street by riding the train in the subway system. When entering the city street, subjects would walk up the subway stairs directly into the street and would enter and exit the airport through the front door. Participants viewed the VR image on a large screen (50 inch $\times 35$ inch) via an LCD projector.

For half the subjects, the airport and the city street were the acquisition and the extinction contexts, respectively. The reverse order was used for the remaining subjects. A high tone $(300 \mathrm{~Hz})$ and a low tone $(135 \mathrm{~Hz})$ delivered via headphones served as CS+ and CS - in a counterbalanced manner. Each tone CS was presented for $8 \mathrm{sec}$. A shock coterminated with the CS+ and no shock was delivered during the CS - . The shock was consistently paired with the CS+ during acquisition only.

An ABA design was used in which subjects were conditioned in context A (e.g., airport), underwent extinction in context B (e.g., city street), and then were returned to context A and B to assess renewal. The experiment was conducted in three phases with a 5-min break between each phase (see Fig. 1). During each phase, a prerecorded scenario was presented. The first phase (3 min $8 \mathrm{sec}$ ) consisted solely of the initial acquisition period in context A. The second phase (15 min $50 \mathrm{sec}$ ) consisted of the second acquisition period in context $\mathrm{A}$ and two extinction periods in context $\mathrm{B}$. The third phase $(10 \mathrm{~min} 52 \mathrm{sec})$ consisted of the final extinction period in context $B$ and a renewal test in context $\mathrm{A}$ and $\mathrm{B}$. Each entry into a context was preceded by a ride in the subway.

During each acquisition period, each CS was presented four times for a total of eight CS+ and eight CS - . During each extinction period, each CS was presented six times for a total of 18 $\mathrm{CS}+$ and $18 \mathrm{CS}-$. The order of presentation of CS+ and CS - was semi-random with the exception that no more that two CS+ or two CS - could be presented consecutively. During the renewal test, which consisted of returning to context A and context B, two CS+ and two CS - were presented in each context. Half of the subjects entered context A followed by context B and half vice versa. For eight subjects, the CS order in each renewal context was $\mathrm{CS}+, \mathrm{CS}-, \mathrm{CS}-$, CS+, and for the remaining subjects, the order was $\mathrm{CS}-, \mathrm{CS}+, \mathrm{CS}+, \mathrm{CS}-$. No shock was administered during extinction or renewal periods.

In order to reduce the number of startle stimuli, no startle stimulus was presented during the first phase. Prior to the second phase, nine startle stimuli were presented in order to habituate the startle reflex. At the start of the second phase, three startle stimuli were delivered in the subway prior to entering context A. Nine startle stimuli were delivered to assess fear conditioning to the CSs during acquisition, one 5-6 sec after the onset of three of the four CS+ and CS-, and three in the absence of CSs (i.e., during ITI). In each extinction period, 15 startle stimuli were delivered, one 5-6 sec after the onset of five of the six CS+ and $\mathrm{CS}-$, and five during ITI. In each context during renewal, six startle stimuli were delivered, one during each of the two CS+ and CS - and two during ITI. The conditioning experiment was designed such that the startle stimuli were presented every 18-26 sec and were delivered at least 10 sec after shock administration to prevent possible influence of the shock sensitization on startle reactivity (Davis 1989).

\section{Procedure}

\section{Conditioning}

Participants were seated $6 \mathrm{ft}$ in front of a screen where the VR image was projected. Electrodes to record the EMG eyeblink and the skin conductance response and to administer the shocks were then attached. A shock work-up procedure was administered to set the shock at an individual level that was moderately painful. Participants were told they were at risk of receiving shock during all three phases and that they may be able to predict the shocks. The experiment was then started. The participants watched all recordings passively in a darkened room.

\section{Subjective anxiety}

Subjective anxiety ratings were obtained only after the renewal test. Subjects were asked to retrospectively rate their overall levels of anxiety the final time they were in each context when tones were played and in the absence of the tones on a scale from 1-10 (1, not at all; 5 , moderately; 10 , extremely).

\section{Data reduction and analysis}

The EMG eyeblink responses were smoothed (20-msec time constant) and rectified, and an onset latency response was deter- 
mined in the 20- to 100-msec time frame after stimulus onset with a peak magnitude no later than $120 \mathrm{msec}$. The baseline EMG recorded $50 \mathrm{msec}$ prior to the startle stimulus onset was then subtracted from the peak magnitude. Eyeblink magnitudes were standardized using within-subjects $T$ scores ([Z scores $X$ $10]+50)$. Skin conductance responses were scored as the largest response 1-4 sec after CS onset. The skin conductance response was determined by subtracting the skin conductance level at the onset of the SCR from the peak skin conductance level. A log transformation $(\log [1+\mathrm{SCR}])$ was performed to normalize the distribution (Venables and Christie 1980).

For acquisition, the eyeblink magnitude and SCR data were averaged within acquisition period 2 in phase 2 . For extinction, the data were averaged over three successive trials resulting in five blocks with eyeblink data (CS+, CS - , ITI) and six blocks of SCR data $(\mathrm{CS}+, \mathrm{CS}-)$. For renewal, the SCR and startle data were averaged over the two CS+ and the two CS - in each context. The blink data were also averaged over the two ITI startle responses in each context.

Statistical analyses were conducted with ANOVAs with repeated measures. To examine acquisition and extinction, the eyeblink magnitude data were entered in a Stimulus Type (CS+, $\mathrm{CS}-$ ) $\times$ Block (one acquisition block and five extinction blocks) ANOVA. A similar ANOVA was implemented for the SCR data with the exception that there were six extinction blocks. For renewal, the order of contexts was not considered in the statistical analysis because preliminary analyses did not show any effect of this variable. The eyeblink magnitude, SCR, and subjective rating data were entered into separate Stimulus Type (CS+, $\mathrm{CS}-$ ) $\times$ Context (Acquisition context, extinction context) ANOVAs. $\alpha$ was set at 0.05 for all statistical tests. GreenhouseGeisser corrections $(G G-\varepsilon)$ were used for main effects and for interactions involving factors with more than two levels.

\section{References}

Baas, J.M.P., Nugent, M., Lissek, S., and Grillon, C. 2003. Context conditioning in a virtual environment potentiates startle. $J$. Psychophysiol. 17 (Suppl. 1): S29.

Baas, J.M., Nugent, M., Lissek, S., Pine, D.S., and Grillon, C. 2004. Fear conditioning in virtual reality contexts: A new tool for the study of anxiety. Biol. Psychiatry 55: 1056-1060.

Bouton, M.E. 2004. Context and behavioral processes in extinction. Learn. Mem. 11: 485-494.

Bouton, M.E. and King, D.A. 1983. Contextual control of the extinction of conditioned fear: Tests for the associative value of the context. $J$. Exp. Psychol. Anim. Behav. Process. 9: 248-265.

Bouton, M.E., Mineka, S., and Barlow, D.H. 2001. A modern learning theory perspective on the etiology of panic disorder. Psychol. Rev. 108: $4-32$.

Branch, M.N. and Hackenberg, T.D. 1998. Humans are animals, too: Connecting animal research to human behavior and cognition. In Learning and behavior therapy (ed. W.T. O'Donohue), pp. 15-35, Allyn \& Bacon, Needham Heights, MA.

Cain, C.K., Godsil, B.P., Jami, S., and Barad, M. 2005. The L-type calcium channel blocker nifedipine impairs extinction, but not reduced contingency effects, in mice. Learn. Mem. 12: 277-284

Craske, M.G., Rowe, M., Lewin, M., and Noriega-Dimitri, R. 1997. Interoceptive exposure versus breathing retraining within cognitive-behavioural therapy for panic disorder with agoraphobia. Br. J. Clin. Psychol. 36: 85-99.

Davis, M. 1986. Pharmacological and anatomical analysis of fear conditioning using the fear-potientiated startle paradigm. Behav. Neurosci. 100: 814-824.

Davis, M. 1989. Sensitization of the acoustic startle reflex by footshock. Behav. Neurosci. 103: 495-503.

Davis, M., Ressler, K., Rothbaum, B.O., and Richardson, R. 2006. Effects of D-cycloserine on extinction: Translation from preclinical to clinical work. Biol. Psychiatry 60: 369-375.

Dawson, M.E. and Furedy, J.J. 1976. The role of awareness in human differential autonomic classical conditioning: The necessary-gate hypothesis. Psychophysiology 13: 50-53.

First, M.B., Spitzer, R.I., Williams, J.B.W., and Gibbon, M. 1995. Structured clinical interview for DSM-V (SCID). American Psychiatric
Association, Washington, DC.

Grillon, C. and Baas, J.M. 2003. A review of the modulation of startle by affective states and its application to psychiatry. Clin. Neurophysiol. 114: $1557-1579$.

Grillon, C. and Davis, M. 1997. Fear-potentiated startle conditioning in humans: Explicit and contextual cue conditioning following paired vs. unpaired training. Psychophysiology 34: 451-458.

Grillon, C., Baas, J.M.P., Cornwell, B., and Johnson, L. 2006. Context conditioning and behavioral avoidance in a virtual reality environment: Effect of predictability. Biol. Psychiatry 60: 752-759.

Hamm, A.O. and Vaitl, D. 1996. Affective learning: Awareness and aversion. Psychophysiology 33: 698-710.

Hermans, D., Dirikx, T., Vansteenwegen, D., Baeyens, F., Van den Bergh, and Eelen, P. 2005. Reinstatement of fear responses in human aversive conditioning. Behav. Res. Ther. 43: 533-551.

Hermans, D., Craske, M.G., Mineka, S., and Lovibond, P.F. 2006. Extinction in human fear conditioning. Biol. Psychiatry 60: 361-368.

Holland, P.C. 1992. Occasion setting in Pavlovian conditioning. In The psychology of learning and motivation, Vol. 28 (ed. D.L. Medin), pp. 69-125. Academic Press, San Diego.

LaBar, K.S. and Phelps, E.A. 2005. Reinstatement of conditioned fear in humans is context dependent and impaired in amnesia. Behav. Neurosci. 119: 677-686.

Lovibond, P.F., Davis, N.R., and O'Flahert, A.S. 2000. Protection from extinction in human fear conditioning. Behav. Res. Ther. 38: 967-983.

Maren, S. and Chang, C.H. 2006. Recent fear is resistant to extinction. Proc. Natl. Acad. Sci. 103: 18020-18025.

Milad, M.R., Orr, S.P., Pitman, R.K., and Rauch, S.L. 2005. Context modulation of memory for fear extinction in humans. Psychophysiology 42: 456-464.

Mineka, S. and Zinbarg, R. 1996. Conditioning and ethological models of anxiety disorders: Stress-in-dynamic-context anxiety models. In Perspective on anxiety, panic, and fear (Nebraska Symposium on Motivation) (ed. D.A. Hope), pp. 135-210. University of Nebraska Press, Lincoln.

Myers, K.M., Ressler, K.J., and Davis, M. 2006. Different mechanisms of fear extinction dependent on length of time since fear acquisition. Learn. Mem. 13: 216-223.

Neumann, D.L.B. 2006. The effects of physical context changes and multiple extinction contexts on two forms of renewal in a conditioned suppression task with humans. Learn. Motiv. 37: 149-175.

Neumann, D.L., Lipp, O.V., and Cory, S.E. 2007. Conducting extinction in multiple contexts does not necessarily attenuate the renewal of shock expectancy in a fear-conditioning procedure with humans. Behav. Res. Ther. 45: 385-394.

Ost, L.G., Brandberg, M., and Alm, T. 1997. One versus five sessions of exposure in the treatment of flying phobia. Behav. Res. Ther. 35: 987-996.

Prokasy, W.F. and Ebel, H.C. 1967. Three components of the classically conditioned GSR in human subjects. J. Exp. Psychol. 73: 247-256.

Quirk, G.J. 2002. Memory for extinction of conditioned fear is long-lasting and persists following spontaneous recovery. Learn. Mem. 9: 402-407.

Rachman, S. and Lopatke, C. 1988. Return of fear: Underlearning and overlearning. Behav. Res. Ther. 26: 99-104.

Thomas, B.L., Larsen, L., and Ayres, J.J.B. 2003. Role of context similarity in $A B A, A B C$, and $A A B$ renewal paradigms: Implications for theories of renewal and for treating human phobias. Learn. Motiv. 34: $410-436$.

Vansteenwegen, D., Hermans, D., Vervliet, B., Francken, G., Beckers, T., Baeyens, F., and Eelen, P. 2005. Return of fear in a human differential conditioning paradigm caused by a return to the original acquistion context. Behav. Res. Ther. 43: 323-336.

Venables, P.H. and Christie, M.J. 1980. Electrodermal activity. In Techniques in psychophysiology (eds. I. Martin and P.H. Venables), pp. 4-67. Wiley, New York.

Vervliet, B., Vansteenwegen, D., Hermans, D., and Eelen, P. 2007. Concurrent excitors limit the extinction of conditioned fear in humans. Behav. Res. Ther. 45: 375-383.

Walker, D.L., Toufexis, D.J., and Davis, M. 2003. Role of the bed nucleus of the stria terminalis versus the amygdala in fear, stress, and anxiety. Eur. J. Pharmacol. 463: 199-216.

Received November 29, 2006; accepted in revised form February 8, 2007. 


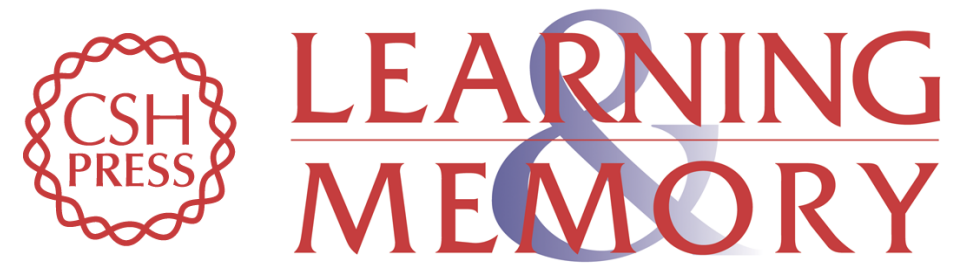

\section{Contextual-specificity of short-delay extinction in humans: Renewal of fear-potentiated startle in a virtual environment}

Ruben P. Alvarez, Linda Johnson and Christian Grillon

Learn. Mem. 2007, 14:

Access the most recent version at doi:10.1101//m.493707

References This article cites 33 articles, 5 of which can be accessed free at:

http://learnmem.cshlp.org/content/14/4/247.full.html\#ref-list-1

License

Email Alerting Receive free email alerts when new articles cite this article - sign up in the box at the Service top right corner of the article or click here. 